\title{
PILOCARPINE DROPS DO NOT REDUCE INTRAOCULAR PRESSURE SUFFICIENTLY IN PSEUDOEXFOLIATION GLAUCOMA
}

\author{
O. BRINCHMANN-HANSEN ${ }^{1}$, T. ALBREKTSEN ${ }^{2}$ and N. ANMARKRUD ${ }^{3}$ \\ Trondheim, Oslo and Lillehammer, Norway
}

\begin{abstract}
SUMMARY
The reason for the poorer prognosis of pseudoexfoliation syndrome glaucomas (PXSG) compared with primary open angle glaucomas (POAG) is not fully understood. An open, comparative, cross-over study was performed in 15 patients (= eyes) with POAG and 15 patients (= eyes) with PXSG. Two different pharmacokinetic principles of drug administration were applied to uncover possible differences in short-term (hours) response to topical antiglaucomatous treatment. Intermittent pilocarpine drop medication (2\%) and continuous low-dose pilocarpine delivery by a membrane-controlled Ocusert unit (P40) were used. The 'carry-over' pressure reduction of an ordinary four times a day drop medication was significantly less effective in controlling the morning intraocular pressure (9 a.m.) in PXSG than in POAG. The duration of action of pilocarpine drops was reduced in PXSG. Defining 'normotensive' pressure as $\leqslant 20 \mathbf{~ m m H g}$, only 1 of the 15 PXSG eyes (6.7\%) reached a normotensive level in the morning, compared with 8 of the 15 POAG eyes (53.3\%). Using a continuous supply of pilocarpine (Ocusert), no differences between POAG and PXSG eyes were found. The study demonstrates the insufficient control of intraocular pressure in PXSG, compared with POAG, by identical antiglaucomatous drop medications. This may suggest an insufficient depot function of topical drugs in PXSG. In consequence, pseudoexfoliation material (PXM) must be sought in eyes with glaucoma, as PXM eyes will probably benefit from a more intense medical treatment compared with eyes without PXM.
\end{abstract}

Glaucoma associated with pseudoexfoliation syndrome has a more serious clinical course than primary open angle

From: ${ }^{1}$ Department of Ophthalmology, Trondheim University Hospital, Trondheim; ${ }^{2}$ Medical Department, Astra Farmasøytiske, Oslo; ${ }^{3}$ Department of Ophthalmology, Lillehammer Central Hospital, Lillehammer, Norway.

Correspondence to: Olaf Brinchmann-Hansen, MD, PhD, Department of Ophthalmology, Ullevaal University Hospital, N-0407 Oslo, Norway. glaucoma (POAG). ${ }^{1-7}$ The reason for this is not fully understood. Pseudoexfoliation syndrome glaucoma (PXSG) is characterised by deposition of fibrillogranular pseudoexfoliative material (PXM) and dispersed pigment in and on intraocular tissues proximating the aqueous humour. The worse prognosis in PXSG could be related to increased resistance to aqueous outflow due to clogging of the trabecular meshwork by PXM, pigment, or both. However, pseudoexfoliation and pigment dispersion may be present in 'normal' eyes in which neither increased intraocular pressure nor glaucoma develop. ${ }^{8-10}$ This suggests that additional mechanisms must, at least in part, account for the difference in outcome. The more frequent need for surgical intervention ${ }^{1,4,5}$ or laser trabeculoplasty ${ }^{11-13}$ in PXSG is often a criterion used in studies which conclude with a difference in prognosis. PXSG seems to respond less satisfactorily than POAG to long-term antiglaucomatous medication, ${ }^{1-3,14-17}$ suggesting a difference in pharmacokinetics in eyes with and without pseudoexfoliation. Possibly, the long-term (years) pressure reducing effects of eye medication wear off more rapidly in PXSG. Alternatively, the short-term (hours) response is less efficient in PXSG, and the cumulative effect of the daily trauma of higher pressures could more easily result in glaucomatous defects in PXSG compared with POAG.

The aim of the present study was to examine whether the short-term response to topical medication differed in the two types of glaucomas. In a cross-over study, we chose pilocarpine because it may be administered to human eyes using two markedly different pharmacokinetic principles: pulsed drop medication and steadystate continuous supply by membrane-controlled delivery (Ocusert P40, Alza).$^{18}$ Since the effect of miotics increases in a systematic manner with increasing pressure, ${ }^{19-21}$ POAG and PXSG eyes were selected on the basis of a protocol allowing for good matching of pre-treatment intraocular pressure levels. 


\section{PATIENTS AND METHODS}

Out-patient Caucasian subjects were recruited, and gave their consent to participate in the study after being informed about its nature and purpose. The criteria for inclusion were as follows: (1) previous diagnosis of open angle glaucoma in one or both eyes; (2) previous treatment with pilocarpine drops; (3) responsiveness to pilocarpine; and (4) after discontinuing pilocarpine for 48 hours, a baseline intraocular pressure (pre-treatment level) $\geqslant 22 \mathrm{mmHg}$ if pilocarpine was the only eye medication or $\geqslant 20 \mathrm{mmHg}$ if additional medication (adrenaline or acetazolamide, or both) was used. Patients were excluded from the study if: (1) there was a history or presence of any internal or external eye diseases; (2) any surgical eye intervention had been performed; (3) the patient used betablocking systemic or local eye medication; and (4) the Ocusert 40 was lost during the test period and not immediately replaced.

Based on inclusion criteria (1) and (2) and exclusion criteria (1), (2) and (3) we recruited patients until 50 eyes with open angle glaucoma had been included. This number of eyes was reached in the first 32 patients and comprised 18 patients with bilateral and 14 patients with unilateral glaucomas. They were subjected to a thorough examination of both eyes which included a slit lamp examination after dilation of the pupils by cyclopentolate (1\%) and metaoxedrin (10\%). We looked for pseudoexfoliation material (PXM) on the lens capsule, and all 50 eyes were classified as either PXM eyes or non-PXM eyes. All patients entered the study and completed the study protocol. During the experiment 2 eyes were excluded due to loss of the Ocusert without its replacement. Two eyes were not responsive to pilocarpine. Forty-eight hours after discontinuing pilocarpine 4 eyes did not reach baseline values above 22 or $20 \mathrm{mmHg}$. Since only one eye from each patient qualified for inclusion, and since comparable pre-treatment intraocular pressures in POAG and PXSG were a presupposition, an additional 12 eyes were excluded. Thus 15 patients with 15 POAG eyes and 15 patients with 15 PXSG eyes entered and completed the study.

\section{Intraocular Pressure}

Goldmann applanation tonometry was carried out for intraocular pressure (IOP) measurements. The mean pressure of three readings was used, and the measurements were performed by alternating between the patient's right and left eye for every reading. This was done whether or not one or both eyes had glaucoma. The mean baseline IOP values \pm standard error of the mean (SE) were $25.8 \pm 1.1 \mathrm{mmHg}$ in POAG $(n=15)$ and $27.7 \pm 1.1$ in PSXG $(n=15)$; the range was $20.3-34.3 \mathrm{mmHg}$. The difference in baseline pressures (mean $1.9 \mathrm{mmHg}$ ) was not statistically significant (confidence interval (CI), 1.13 to $5.01 ; \mathrm{SE}, 1.50 ; p<0.20$ ).

\section{Study Protocol}

The study was performed as an open, comparative and controlled study, and each patient was subjected to two experimental sessions. The patients were randomly allocated to start either with (1) pilocarpine (2\%) drop medication or (2) Ocusert placed in either the upper or lower cul-de-sac. Each treatment session was preceded by a 44-48 hour wash-out period without pilocarpine; an IOP reading at 9 a.m. after 24 hours of wash-out was taken as the pre-treatment baseline value. The next day (day 1) patients assigned to drops started the medication using one drop four times daily ( 9 a.m., 1 p.m., 5 p.m. and 9 p.m.), while the other group received the Ocusert unit at 9 a.m. In both treatment groups the IOP was measured the following morning (day 2) at 9 a.m. Thereafter, the drop treatment group was given (by us) one drop of pilocarpine, while the Ocusert group continued wearing the unit. The IOP was again measured in all patients at 11 a.m. and 1 p.m. After having completed either the drop or the Ocusert session, pilocarpine was again discontinued for another 44-48 hour wash-out period. The patients (eyes) then crossed over to the other mode of pilocarpine administration, and an identical treatment session was repeated.

\section{Statistical Analysis}

Student's $t$-test for paired comparison was used within groups and an independent $t$-test for testing differences between administration forms. We defined 'normotensive pressure' as $\leqslant 20 \mathrm{mmHg}$, and the difference between groups regarding normotension was calculated using Fisher's exact test. Spearman's correlation coefficient was used when studying the relation between the reduced IOP and the pre-treatment level. Statistical significance was accepted at the 5\% level (two-sided).

\section{Clinical Background Data}

Age ranged from 44 to 80 years (mean 64.9 years) in POAG patients (10 men, 5 women) and from 54 to 88 years (mean 73.7 years) in PXSG patients (6 men, 9 women). All patients were native Norwegians and had basically blue irides; their refractive errors ranged from -3.0 to +2.0 dioptres. The anterior chamber angle width and the trabecular pigmentation were registered by gonioscopy at 12, 3, 6 and 9 o'clock, and a mean score was given for each eye. A 4-point scale was used: ${ }^{22}$ (1) narrow angle, extreme; (2) narrow angle, moderate; (3) open angle; and (4) wide open angle. The angle width ranged from 2 to 4, averaging 3.3 for POAG eyes and 3.2 for PXSG eyes. The trabecular pigment band was graded on a 4-point scale:22 (1) slight pigmentation; (2) moderate/ slight; (3) moderate/dense; and (4) dense dark pigmentation band. Pigmentation ranged from 1 to 4 , averaging 1.8 for POAG eyes and 2.6 for PXSG eyes. The cup/disc ratio as subjectively determined by ophthalmoscopy ranged from 0.1 to 1.0 , averaging 0.7 in both POAG and PXSG eyes. Nerve fibre defects was registered by Goldmann perimetry for visual field examination using one isopter, and were observed in 5 POAG and 6 PXSG eyes. The defects ranged from a wide blind spot to loss of no more than a nasal quadrant. Adrenaline 1\% twice a day was used by 4 

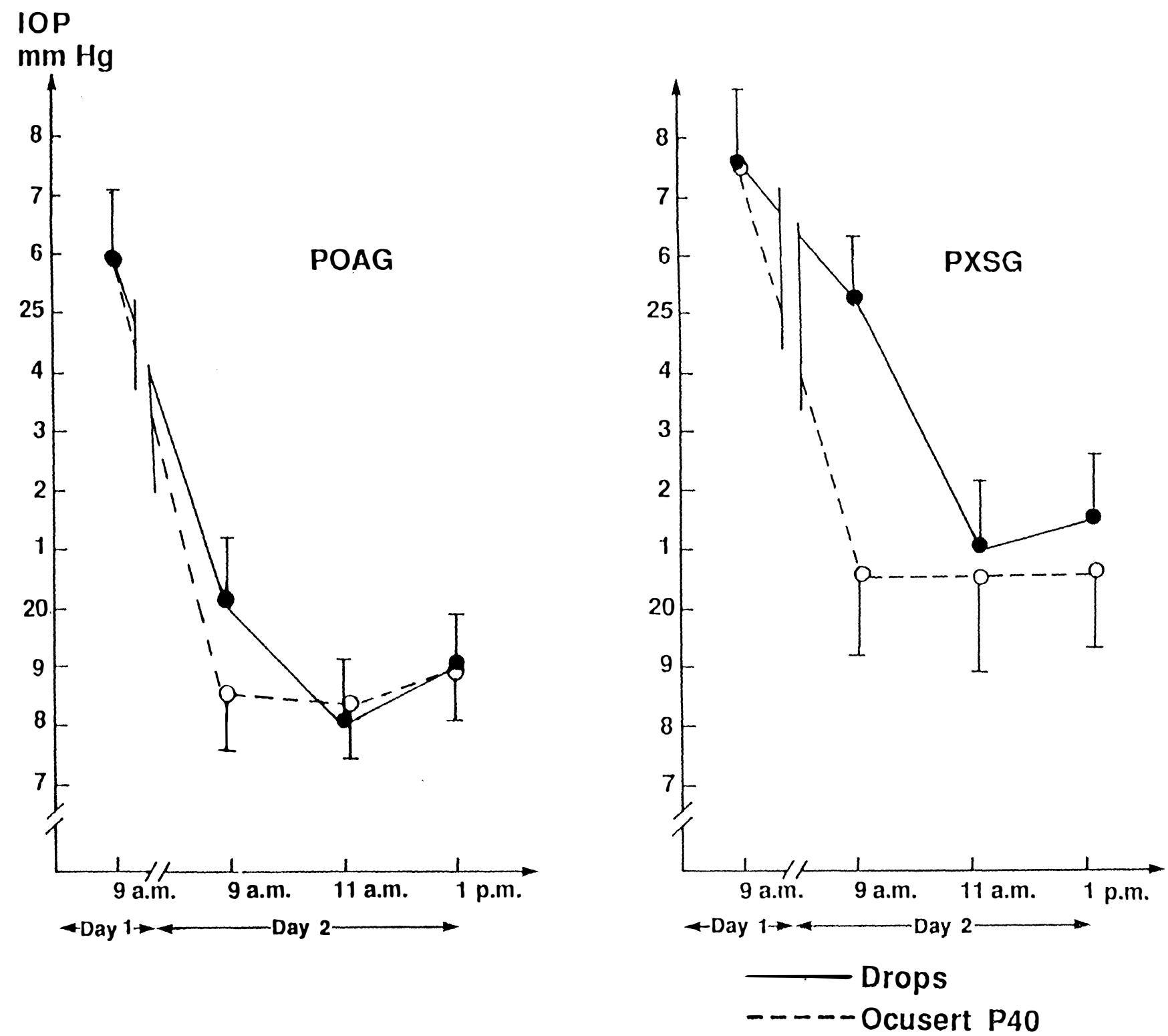

Fig. 1. Pressure reducing effects of drop medication and continuous delivery (Ocusert P40) of pilocarpine in comparable groups of patients with primary open angle glaucomas (POAG) and pseudoexfoliation syndrome glaucomas (PXSG). Both treatment regimens in $P O A G$ and Ocusert in PXSG on day 1 'carried over' pressure reducing effects on 9 a.m. on day 2 ( $\mathrm{p}<0.005-0.001)$. Drop medication on day 1 in PXSG was insufficient for pressure control at 9 a.m. on day 2 in PXSG, the difference in the response to drops between $P O A G$ and PXSG being statistically significant $(\mathrm{p}<0.05)$.

'eyes' with POAG and 7 eyes with PXSG, while acetazolamide $250 \mathrm{mg}$ twice daily was used by 3 POAG and 3 PXSG eyes. These medications were continued unchanged during the study.

\section{RESULTS}

Fig. 1 gives the mean IOP \pm SE of the 15 POAG and 15 PXSG eyes using pilocarpine drop medication and the Ocusert unit. The pressure reducing effect of Ocusert was greater than that of pilocarpine drops at 9 a.m. (day 2) in both types of glaucoma, $(\mathrm{mmHg}( \pm \mathrm{SE}), \mathrm{CI})$ : in POAG 1.7 ( \pm 0.8$), 0.01$ to $3.43(p<0.05)$; and in PXSG 4.5 ( \pm $1.5), 1.34$ to $7.74(p<0.01)$ (Fig. 1).

Furthermore, Fig. 1 shows that the 9 a.m. drop of pilocarpine on day 2 further reduced IOP to a significantly lower level in POAG $(p<0.005)$ and PXSG $(p<0.02)$ through 11 a.m. and 1 p.m. No differences between Ocusert and pilocarpine drops were observed at 11 a.m. or 1 p.m. in either type of glaucoma.

Table I shows that pilocarpine drops and Ocusert significantly reduced the 9 a.m. (day 2 ) pressure in both types of glaucoma. The pressure reduction achieved with the Ocusert was no different in POAG and PXSG (7.2 mmHg and $7.1 \mathrm{mmHg}$, respectively), while the 'carry-over' pressure lowering effect of pilocarpine drops was significantly smaller in PXSG $(0.1 \mathrm{mmHg})$ than in POAG $(3.0 \mathrm{mmHg}$; $p<0.05$ ) (Table I).

Fig. 2 illustrates the percentage of eyes in which IOP was lowered to a 'normotensive' level $(\leqslant 20 \mathrm{mmHg})$ at 9 a.m. (day 2) in POAG and PXSG using either pilocarpine drops or Ocusert. The number of normotensive POAG eyes, using pilocarpine drops and Ocusert, was 8 of 15 
Table I. Mean differences $( \pm$ SE) in pressure reduction at 9 a.m. (day 2) between pilocarpine drops and Ocusert in POAG and PXSG

\begin{tabular}{lccc}
\hline & IOP reduction & SE & $p$ value \\
\hline $\begin{array}{l}\text { Pilocarpine drops } \\
\text { POAG (mmHg) }\end{array}$ & 5.5 & \pm 1.2 & $<0.001$ \\
PXSG (mmHg) & 2.5 & \pm 0.7 & $<0.005$ \\
Difference & 3.0 & \pm 1.4 & $<0.05$ \\
Ocusert & & & \\
POAG (mmHg) & 7.2 & \pm 1.2 & $<0.001$ \\
PXSG & 7.1 & \pm 1.3 & $<0.001$ \\
$\quad$ Difference & 0.1 & \pm 1.8 & NS $^{\mathrm{a}}$ \\
\hline
\end{tabular}

${ }^{a} 95 \%$ confidence intervals were: pilocarpine drops, 0.05 to 5.85 ; Ocusert, -3.49 to 3.75 .

(53.3\%) and 11 of 15 (73.3\%), respectively; the difference $(20 \%)$ between the two modes of treatment was not statistically significant $(\mathrm{CI}-13.7 \%$ to $53.7 \% ; p<0.16)$. The number of normotensive PXSG eyes, using pilocarpine drops and Ocusert, was 1 of $15(6.7 \%)$ and 8 of 15 (53.3\%), respectively; the difference $(47 \%)$ was statistically significant (CI $18.4 \%$ to $75.0 \% ; p<0.007$ ).

The PXSG patients were somewhat older (74 years) than the POAG patients (65 years), and we sought a possible relation between age and mean IOP reduction. We analysed this relation from baseline to 9 a.m. on day 2, after the drop medication had been performed by the patients themselves, and at 11 a.m. on day 2 after the pilocarpine drop had been instilled by one of the authors (O.B.-H.). Including all 30 eyes, the regression lines (Pearson's) $y=a x+b$, where $y$ is the reduction in IOP and $x$ is age) were $y=0.01 x+3.4$ and $y=0.009 x+2.4$, respectively, i.e. no relation between age and reduction in IOP was found in our patients.

\section{DISCUSSION}

The hypothetical rationale for the design of the present study was that PXM or abnormalities of pigment distribution could interact with intraocular drug absorption or depot function, or inactivate pulsed drop medication ( = abnormal drug metabolism). If this explanation were valid, the worse prognosis in PXSG could be caused by complete or partial loss of pressure control in the 12 hours (9 p.m. to 9 a.m.) between drop medications in PXSG but not in POAG. This was found to be the case in the present study.

Furthermore, a continuous supply of pilocarpine to presumed normal pressure reducing 'receptors' could potentially 'short-cut' the abnormal drug metabolism in PXSG. If this explanation were valid, Ocusert should be capable of pressure reduction of similar strength in both PXSG and POAG. This was also found to be the case in the study.

Accordingly, the results suggest a difference in the pharmacokinetics of pilocarpine drop medication in the two types of glaucomas, affecting either absorption, depot function or inactivation of the drug. Absorption of the drug may not be the main problem since the maximum pressure reducing response was not very different in PXSG and POAG. Furthermore, the sensitivity of the pressure reducing 'receptors' may not be different in the two types of glaucomas: a $7.2 \mathrm{mmHg}$ and a $7.1 \mathrm{mmHg}$ pressure reduction were obtained in POAG and PXSG, respectively, both responding to a continuous supply of pilocarpine (Ocusert unit).

The depot function of pilocarpine in intraocular tissues is well documented. The duration of the pressure reducing effect of a single drop of medication ${ }^{23}$ exceeds, by far, the time when pilocarpine is present in the aqueous. ${ }^{24}$ Sendelbeck et al. ${ }^{25}$ studied the distribution of pilocarpine in different ocular tissues in rabbits using both drop medication and an Ocusert unit. They found that the depot levels in cornea, iris and sclera 3-6 hours after drop administration were approximately equal to those maintained by the Ocusert unit. This coincides with the pressure reducing effects found 2 and 4 hours after drop instillation. The Ocusert and the pilocarpine drop were equally effective in

\section{Normotensive eyes \\ $\%$ \\ (9 a.m.)}

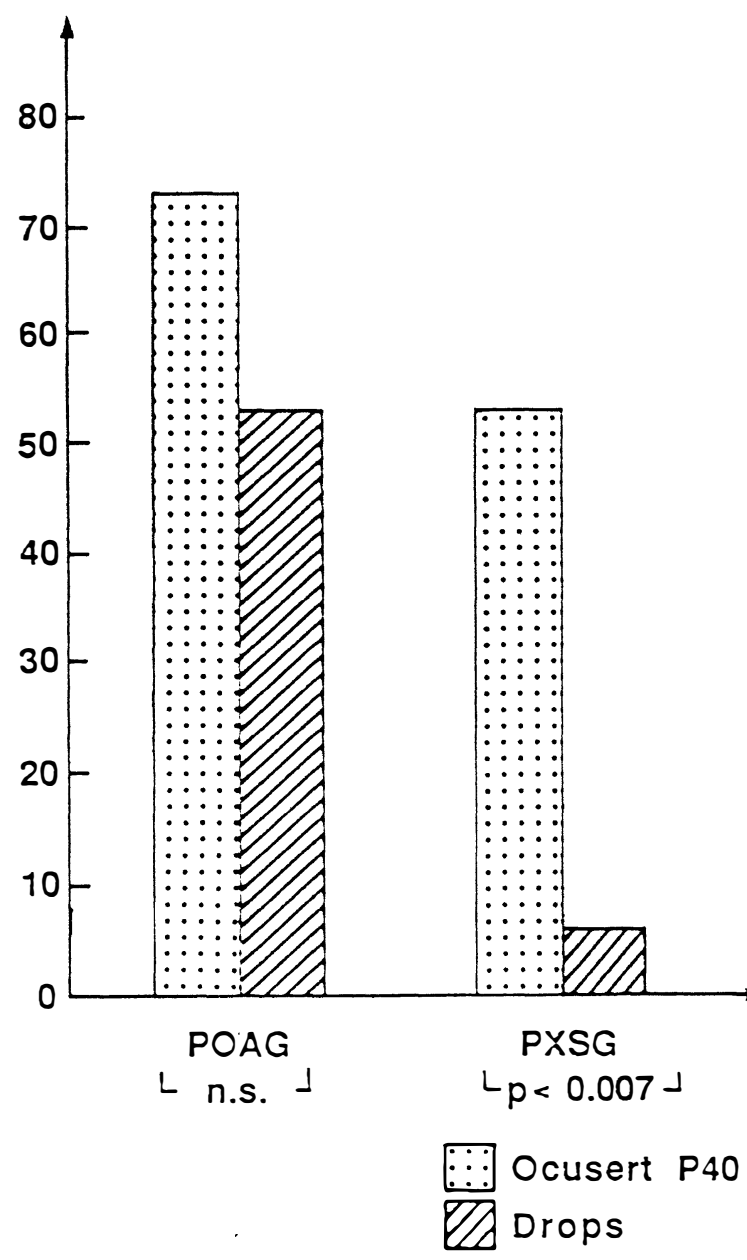

Fig. 2. The percentage of normotensive eyes $(\leqslant 20 \mathrm{mmHg}$ ) registered at 9 a.m. (day 2) in POAG and PXSG using either pilocarpine drops or Ocusert P40. Only 1 of 15 PXSG eyes (6.7\%) reached a normotensive level at 9 a.m. on drop medication, compared with 8 of 15 POAG eyes $(53.3 \%)(\mathrm{p}<0.007)$. Eleven of 15 $P O A G$ eyes $(73.3 \%)$ were normotensive on a continuous supply of pilocarpine from the Ocusert. 
keeping the pressure low at 11 a.m. and 1 p.m., in both POAG and PXSG eyes.

Correspondingly, we feel it can be assumed that the latest pilocarpine drop (9 p.m. on day 1) in a normal four times a day regime was equally effective in both types of glaucomas through the first 4 hours of the night interval. The 'carry-over' effect through late night and early morning was, however, significantly smaller in PXSG, suggesting a shorter intraocular duration of effect of the drop medication.

We may speculate why this was the case. Melanin is a specific binding site for drug absorption, ${ }^{26,27}$ and heavy ocular pigmentation somehow binds topically administered drug, ${ }^{28-32}$ prolonging the duration of action of a drop medication. Possibly the pigment dispersion and loss of iris pigment, or the PXM distribution, of PXSG eyes reduce the 'normal' quantity of depots or, less likely, increase the drug elimination rate (inactivation). Experimental studies in rabbits ${ }^{33,34}$ suggest that pilocarpine medication affects blood flow in the iris and ciliary processes. Perhaps blood flow in the anterior segments of POAG and PXSG eyes is basically different, or differently affected by pilocarpine, resulting in differences in depot function or inactivation of the drug, or in production of aqueous.

An insufficient response to topical medication may thus contribute to the difference in prognostic outcome, but additional factors exist. There seems to be an increased tendency in PXSG relative to POAG to develop glaucomatous damage at any given level of IOP ${ }^{35}$ It is not known why this is the case. Furthermore, higher IOP values are observed in PXSG compared with POAG,${ }^{1,8,9}$ and this certainly could affect the prognosis.

In summary, the present study shows that the 'carryover' pressure reducing effect of continuous delivery of pilocarpine (Ocusert unit), through late night and early morning, is superior to drop medication in both POAG and PXSG eyes. The duration of action of pilocarpine drops is significantly shorter in PXSG eyes than in POAG eyes. In consequence only 1 of 15 PXSG eyes $(6.7 \%)$ was found to be 'normotensively' controlled by pilocarpine drops in the morning, compared with 8 of 15 POAG eyes (53.3\%). An association between IOP peaks and progression of visual field loss has been established, ${ }^{36}$ and peaks of higher IOP pressure are probably more frequent in PXSG than in POAG. Recent studies suggest that darkness and sleep ${ }^{37,38}$ and waking in the morning ${ }^{39}$ significantly increase IOP. The observed difference in morning IOP seems of a magnitude large enough to suggest continuous daily trauma to nerve fibres, the cumulative effect of which is a worse long-term prognosis of PXSG.

Key words: Glaucoma capsulare, Intraocular pressure, Open angle glaucoma, Pharmacokinetics, Pilocarpine, Pseudoexfoliation.

\section{REFERENCES}

1. Tarkkanen A. Treatment of chronic open-angle glaucoma associated with pseudoexfoliation. Acta Ophthalmol (Copenh) 1965;43:514-23.

2. Hørven I. Exfoliation syndrome: incidence and prognosis of glaucoma capsulare in Massachusetts. Arch Ophthalmol 1966;76:505-11.
3. Klouman OF. Pseudoexfoliation in ophthalmic practice. Acta Ophthalmol (Copenh) 1967;45:822-8.

4. Aasved $\mathrm{H}$. The frequency of optic nerve damage and surgical treatment in chronic simple glaucoma and capsular glaucoma. Acta Ophthalmol (Copenh) 1971;49:489-600.

5. Layden WE, Shaffer RN. Exfoliation syndrome. Am J Ophthalmol 1974;78:835-41.

6. Olivius E, Thorburn W. Prognosis of glaucoma simplex and glaucoma capsulare: a comparative study. Acta Ophthalmol (Copenh) 1978;56:921-34.

7. Brooks AMV, Gillies WE. The presentation and prognosis of glaucoma in pseudoexfoliation of the lens capsule. Ophthalmology 1988;95:271-6.

8. Hansen E, Sellevold OJ. Pseudoexfoliation of the lens capsule. III. Ocular tension in eyes with pseudoexfoliation. Acta Ophthalmol (Copenh) 1970;48:446-54.

9. Aasved H. Intraocular pressure in eyes with and without fibrillopathia epitheliocapsularis (so-called senile exfoliation or pseudoexfoliation). Acta Ophthalmol (Copenh) 1971;49:601-10.

10. Bedikt O, Roll P. The trabecular meshwork of a non-glaucomatous eye with the exfoliation syndrome. Virchows Arch [A] 1979;384:347-55.

11. Pohjanpelto P. Argon laser treatment of the anterior chamber angle for increased intraocular pressure. Acta Ophthalmol (Copenh) 1981;59:211-5.

12. Ritch R, Podos SM. Laser trabeculoplasty in the exfoliation syndrome. Bull NY Acad Med 1983;59:339-44.

13. Tuulonen A, Airaksinen PJ. Laser trabeculoplasty in simple and capsular glaucoma. Acta Ophthalmol (Copenh) 1983;61:1009-15.

14. Kerty E, Hørven I. Glaucoma treatment with timolol. Acta Ophthalmol (Copenh) 1978;56:705-13.

15. Airaksinen PJ. The long-term hypotensive effect of timolol maleate compared with the effect of pilocarpine in simple and capsular glaucoma. Acta Ophthalmol (Copenh) 1979; 57:425-34.

16. Aasved H, Seland JH, Slagsvold JE. Timolol maleate in treatment of open-angle glaucoma. Acta Ophthalmol (Copenh) 1979;57:700-8.

17. Blika S, Saunte E. Timolol maleate in the treatment of glaucoma simplex and glaucoma capsulare. Acta Ophthalmol (Copenh) 1982;60:967-76.

18. Armaly MF, Rao K. The effect of pilocarpine Ocusert on ocular pressure. In: Leopold IH, editor. Symposium of ocular therapy. Vol. 6. St. Louis: Mosby, 1973:80-94.

19. Davanger M. The pressure reducing effect of miotics in glaucoma simplex and in normal eyes, elucidated by hydrodynamic calculations based on Poiseuille's law. Acta Ophthalmol (Copenh) 1964;42:773-81.

20. Krill AE, Newell FW. Effects of pilocarpine on ocular tension dynamics. Am J Ophthalmol 1964;57:34-41.

21. Brinchmann-Hansen O, Anmarkrud N. Pilocarpine medication in open-angle glaucoma. Acta Ophthalmol (Copenh) 1979;57:55-62.

22. Kolker AE, Hetherington J Jr. Clinical interpretation of gonioscopic findings. In: Becker-Schaffer's diagnosis and therapy of the glaucomas. 4th ed. St. Louis: Mosby, 1976:42-53.

23. Drance SM, Bensted M, Schulzer M. Pilocarpine and intraocular pressure. Arch Ophthalmol (Copenh) 1974;91: 104-6.

24. Asseff CF, Weisman RL, Podos SM, Becker B. Ocular penetration of pilocarpine in primates. Am $\mathrm{J}$ Ophthalmol 1973;75:212-5.

25. Sendelbeck L, Moore D, Urquhart J. Comparative distribution of pilocarpine in ocular tissues of the rabbit during administration by eyedrop or by membrane-controlled delivery systems. Am J Ophthalmol 1975;80:274-83.

26. Havener WH. Autonomic drugs. In: Ocular pharmacology. 4th ed. St. Louis: Mosby, 1978:267-87. 
27. Shell JW. The pharmacokinetics of topical glaucoma medications. In: Drance SM, Neufeld AH, editors. Glaucoma: applied pharmacology in medical treatment. New York: Grune \& Stratton, 1984:185-213.

28. Melikian HE, Lieberman TW, Leopold IH. Ocular pigmentation and pressure and outflow responses to pilocarpine and epinephrine. Am J Ophthalmol 1971;72:70-3.

29. Harris LS, Galin MA. Effect of ocular pigmentation on hypotensive response to pilocarpine. Am J Ophthalmol 1971;72:923-5.

30. Lyons JS, Krohn DL. Pilocarpine uptake by pigmented uveal tissue. Am J Ophthalmol 1973;75:885-8.

31. Salminen L, Imre G, Huupponen R. The effect of ocular pigmentation on intraocular pressure response to timolol. Acta Ophthalmol (Copenh) 1985;63 (Suppl 173):15-8.

32. Abrahamsson T, Bostrøm S, Brautigam J, Lagerstrøm PO, Regårdh CG, Vauqelin G. Binding of the beta-blockers timolol and H 216/44 to ocular melanin. Exp Eye Res 1988;47:565-77.

33. Alm A, Bill A, Young FA. The effects of pilocarpine and neostigmine on the blood flow through the anterior uvea in monkeys: a study with radioactively labelled microspheres. Exp Eye Res 1973;15:31-6.

34. Green K, Hatchett TL. Regional ocular blood flow after chronic topical glaucoma drug treatment. Acta Ophthalmol (Copenh) 1987;65:503-6.

35. Davanger M, Ringvold A, Blika S. Pseudo-exfoliation, IOP and glaucoma. Acta Ophthalmol (Copenh) 1991;69:569-73.

36. Zeimer RC, Wilensky JT, Gieser DK, Viana MAG. Association between intraocular pressure peaks and progression of visual field loss. Ophthalmology 1991;98:64-9.

37. Birchely A, Mudie P, Brown B. IOP elevation in different phases of sleep. Clin Exp Optom 1990;73:93-6.

38. Wildsoet CF, Brown B, Swann PG. Darkness and sleep as contributing factors to diumal variation in intraocular pressure. Glaucoma 1990;12:140-7.

39. Zeimer RC, Wilensky JT, Gieser DK. Presence and rapid decline of early morning intraocular pressure peaks in glaucoma patients. Ophthalmology 1990;97:547-50. 